\title{
Acute pancreatitis and vasoplegic shock associated with leptospirosis - a case report and review of the literature
}

\author{
Alexander Maier ${ }^{1,2}$, Rafael Kaeser ${ }^{2}$, Robert Thimme ${ }^{2}$ and Tobias Boettler ${ }^{2^{*}}$ (D)
}

\begin{abstract}
Background: Leptospirosis or Weil's disease is caused by pathogenic spirochete bacteria called Leptospira. It is considered the most common zoonosis in the world and is usually transmitted by urine of rodents and dogs with an incubation time of 7-14 days. The clinical spectrum ranges from a subclinical infection to a fulminant septic course.

Case presentation: Here, we report the case of a German patient with acute pancreatitis associated with Leptospira interrogans causing fulminant septic shock. The patient was successfully treated with intravenous antibiotics and left the hospital fully recovered after 18 days.

Conclusions: To our knowledge, this is the first case of leptospirosis with acute pancreatitis as the leading clinical manifestation in Central Europe. Serologic and molecular genetic tests for leptospirosis should be considered, if no other causes for pancreatitis can be identified.
\end{abstract}

Keywords: Leptospirosis, Pancreatitis, Septic shock, Europe, Case report

\section{Background}

Leptospirosis or Weil's disease is caused by pathogenic spirochete bacteria called Leptospira. It is considered to be the most common zoonosis worldwide and is usually transmitted from rodents, dogs and cats when an infected animal or an animal's urine comes into contact with broken skin. The incubation time is 7-14 days. Absence of infected skin makes the entry unidentifiable in most cases. The clinical spectrum ranges from a subclinical infection to a fulminant septic course. Typical symptoms are shivering, fever, myalgia, conjunctival suffusion, vomiting and diarrhea. Icteric and unicteric courses are known. Jaundice is considered as a sign of liver dysfunction but can occur without necrosis of the liver. Antibiotic treatment must be initiated early. Most cases of leptospirosis are described from tropical or subtropical regions. In Germany, approximately 60 cases occur each year [1]. Here, we report the case of a German patient, who presented with acute pancreatitis

\footnotetext{
*Correspondence: tobias.boettler@uniklinik-freiburg.de

${ }^{2}$ Department of Medicine II, Medical Center, Faculty of Medicine, University

of Freiburg, Hugstetter Strasse 55, 79106 Freiburg, Germany

Full list of author information is available at the end of the article
}

associated with Leptospira interrogans causing fulminant septic shock.

\section{Case presentation}

Five days prior to hospital admission, the 73-year old male suffered from mucous congestion, a swollen throat, fever and shivering. He subsequently developed severe leg-weakness, back pain and jaundice as well as psychiatric symptoms with delusion and hallucination while his fever worsened with a body temperature of $39.8^{\circ} \mathrm{C}$. Myalgia did not occur initially. After consulting his primary care physician, he was immediately transferred to the emergency department (ED) at another hospital.

Apart from a history of depression and Helicobacter pylori - gastritis a few years ago, his medical history was unremarkable with no other preexisting conditions. There was no recent travel nor alcohol consumption. The only regular medication was $40 \mathrm{mg}$ of Citalopram daily. The physical examination revealed jaundice and minor petechiae at the lower legs. The abdomen was slightly distended with pain after palpation in the right upper quadrant. Peristaltic sounds were normal. Examination of the heart and the lungs were unremarkable. Conjunctival suffusion was not found. 
Initial blood analysis revealed leukocytosis (13.2/nl), thrombocytopenia $(23 / \mathrm{nl})$ as well as elevated values for C-reactive protein (CRP, $174.6 \mathrm{mg} / \mathrm{l}$ ), procalcitonin (PCT, $10.0 \mathrm{ng} / \mathrm{ml}$, cutoff $<0.5 \mathrm{ng} / \mathrm{ml})$, lactate dehydrogenase $(\mathrm{LDH})(312 \mathrm{U} / \mathrm{l})$, creatinine $(2.95 \mathrm{mg} / \mathrm{dl}$, clearance $20 \mathrm{ml} /$ $\mathrm{min} / 1.73 \mathrm{qm}$ ), aspartate aminotransferase (AST, $55 \mathrm{U} / \mathrm{l}$ ), bilirubin $(14.9 \mathrm{mg} / \mathrm{dl}$, direct bilirubin $11,72 \mathrm{mg} / \mathrm{dl})$ and lipase $(2417 \mathrm{U} / \mathrm{l})$. Sodium $(125 \mathrm{mmol} / \mathrm{l})$ and potassium $(3.18 \mathrm{mmol} / \mathrm{l})$ were reduced, hemoglobin $(\mathrm{Hb})$, alkaline phosphatase (AP), gamma-glutamyltransferase (GGT) and alanine aminotransferase (ALT) were normal. An overview of selected initial laboratory values can be found in Table 1. Urine analysis revealed nitrite positivity with leukocytes, proteinuria, erythrocytes and bilirubin. An initial X-ray of the chest showed no relevant pathologies.

Due to acute septic pancreatitis and acute kidney injury he was transferred to the intensive care unit (ICU). Antibiotic treatment was started with ampicillin/sulbactam. Although no cholestasis or gallstones were found in an initial abdominal ultrasound, a biliary pancreatitis with sepsis was suspected. Thus, an endoscopic retrograde cholangiopancreatography (ERCP) was performed 1 day after his primary presentation at the ED. This procedure revealed a normal duodenum, a swollen papilla vateri and only minor spontaneous biliary drainage was observed. Intrahepatic bile ducts and the common hepatic duct were not dilated. While preparing for the endoscopic procedure, the respiratory and hemodynamic

Table 1 Selected initial laboratory values

\begin{tabular}{ll}
\hline Leukocytes [4.0-10.0] [/nl] & 13.2 \\
Hemoglobin [13.5-17.5] [g/dl] & 14.0 \\
Hematocrit [40-53] [\%] & 42.0 \\
Thrombocytes [140-360] [/nl] & 23 \\
Sodium [135-144] [mmol/l] & 125 \\
Potassium [3.50-5.10] [mmol/l & 3.18 \\
Urea [17-43] [mg/dl] & 139 \\
Creatinine [0.7-1.4] [mg/dl] & 2.95 \\
AP [40-129] [IU/l] & 69 \\
ALT (GPT) [< 45] [IU/l] & 36 \\
AST (GOT) [< 35] [IU/l] & 55 \\
Y-GT [< 60] [IU/l] & 25 \\
Bilirubin [< 1.20] [mg/dl] & 14.90 \\
Direct bilirubin [< 0.3] [mg/dl] & 11.72 \\
Indirect bilirubin [< 1.20] [mg/dl] & 3.18 \\
Lipase [73-393] [IU/l] & 2417 \\
LDH [< 248] [IU/l] & 312 \\
CRP [< 5] [mg/l] & 174.6 \\
PCT [< 0.5] [ng/ml] & 10.0 \\
\hline
\end{tabular}

condition of the patient worsened. Consequently he was intubated, commenced on a noradrenaline infusion, and the antibiotic treatment was escalated to meropenem.

A chest CT scan demonstrated bilateral pulmonary infiltrates and small bilateral pleural effusions. Microbiological analysis of blood cultures was negative at all time points tested. A screening for ANA and ANCA was also negative.

The following day, the patient was transferred to our university medical center ICU with sepsis and acute pancreatitis, acute kidney failure and acute respiratory distress syndrome (ARDS).

In our ICU, antibiotic treatment with meropenem and catecholamine treatment with noradrenaline were continued. Blood cultures were taken repetitively and a sample of the tracheal secretion was obtained. After 5 days, weaning from artificial respiration and noradrenaline was possible and the patient was transferred to our gastroenterological ward. The physical examination revealed a slight transient rash of his back. We performed further investigations regarding the etiology of the patient's condition. A stool test for Clostridium difficile antigen was negative. Further sampling by blood cultures, tracheal secretion and urine-analyses showed no growth of any bacteria. A screening for MRSA was negative, as were serologic tests for Hepatits B, Hepatitis $\mathrm{C}$, mumps and Epstein-Barr virus. Acute liver failure with jaundice, hemorrhagic diathesis with purpura and petechiae combined with acute kidney injury accompanied by an odd transient macular, erythematous rash directed to serologic testing for leptospirosis. It revealed strong positive IgG and IgM antibodies. While leptospirosis PCR in the blood was negative, analysis of the tracheal secretion taken during his stay on the ICU revealed the presence of Leptospira interrogans DNA. Urine analysis performed at the same time however remained negative for leptospirosis DNA by PCR. PCR was performed by the Institute of Microbiology and Hygiene of the University of Freiburg, Germany, which is accredited by the DAkkS (Deutsche Akkreditierungsstelle, Berlin, Germany) for leptospira PCR for probes of both sterile and non-sterile origin.

An ultrasound of the patient demonstrating prominent edematous pancreas can be found in Fig. 1.

The patient was discharged 18 days after his atypical presentation at the ED with normalized lab values.

\section{Discussion and conclusion}

Here, we report of a patient who presented with acute pancreatitis followed by septic shock with ARDS and acute kidney injury that was caused by leptospirosis. The patient also had mild hepatitis at presentation with clinical jaundice, elevated AST and bilirubin; however, ALT 


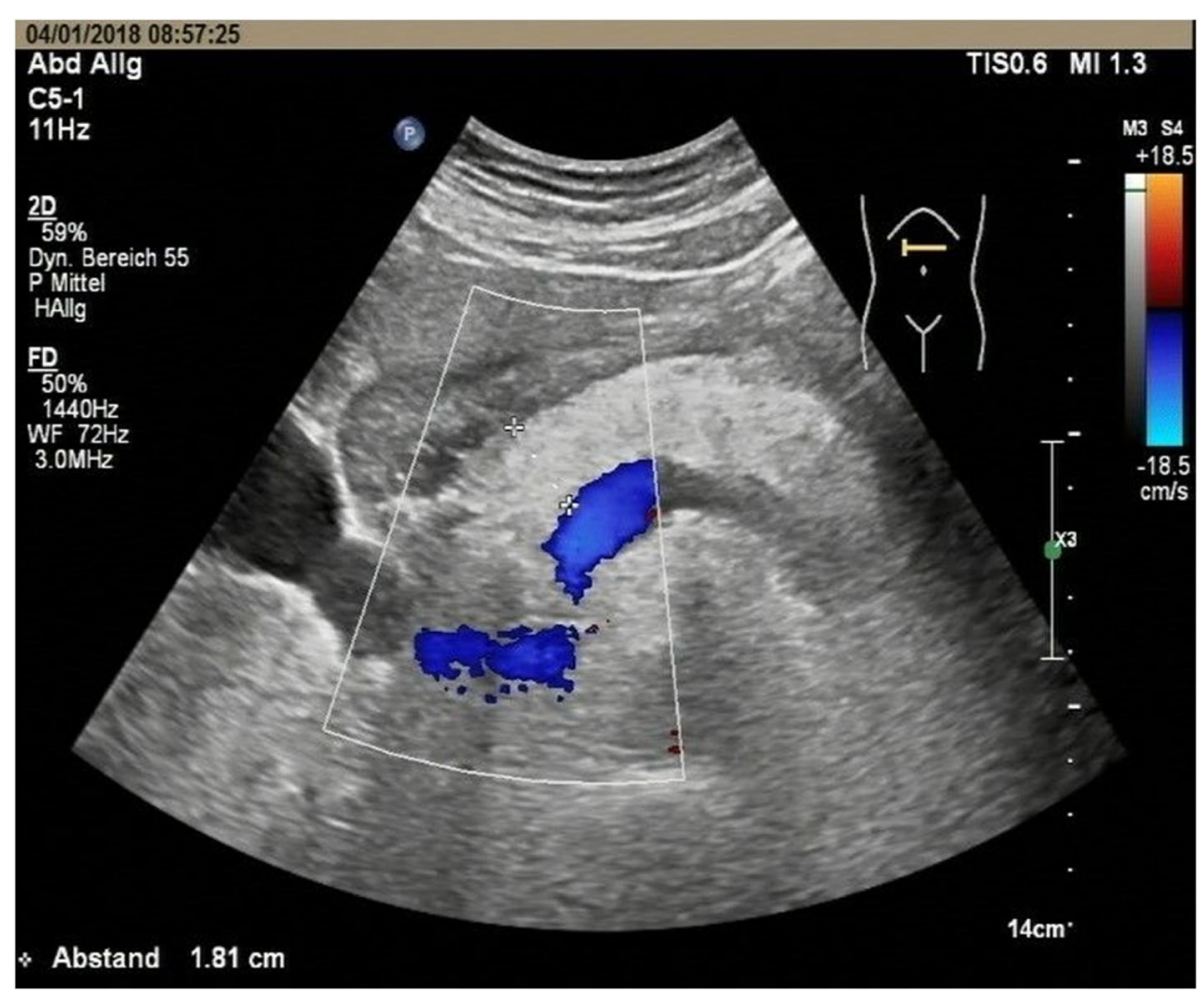

Fig. 1 Ultrasound of the patient demonstrating prominent edematous pancreas

values were not increased throughout the hospital stay and AST levels decreased to normal within 5 days.

The source of his infection eventually remains unclear. There are two relevant possibilities which appear to be most likely: First, the patient lives with several pets including two cats and a dog, which might have shed leptospires in the urine [2]; second, he visited a waste deposal site 7 days before his initial presentation at the ED but had never noticed contact with rodents or recognized a bite injury.

To our knowledge, this is the first case in Central Europe with acute pancreatitis causing vasoplegic shock by leptospirosis. Few cases with leptospirosis have been described in South Europe: One with pancreatitis and cholecystitis in Greece in 2004 [3]. Another case from Greece about a patient from Congo reported pancreatitis in combination with myocarditis, polyarthritis, mononeuritis multiplex and vasculitis with symmetrical peripheral gangrene of the lower extremities [4]. A similar case from Romania was reported in 2013 with necrotizing pancreatitis and severe anemia which required surgical therapy [5]. Some cases with leptospirosis leading to pancreatitis have been described in tropical or subtropical regions [6-18]. An overview of the previously described cases of leptospirosis and pancreatitis can be found in Table 2. However, diagnosis of pancreatitis was often based on amylase-levels [4, 6, 12-14, 18].
These cases have to be interpreted with caution as amylase is often increased in patients with renal failure which is one of the most common manifestations of leptospirosis $[19,20]$. Analysis of pancreatic lipase should be preferred as it is more specific for the diagnosis of pancreatitis.

In Germany, doxycycline, penicillin G, cefotaxime, and ceftriaxone are recommended by the Robert-Koch-Institute as first-line agents for the treatment of leptospirosis. Our patient was initially treated with ampicillin/sulbactam which was then escalated to meropenem. A specific antibiotic treatment like doxycycline, penicillin or ceftriaxone was not administered because of the late diagnosis of leptospirosis.

It is especially peculiar and remarkable that in this patient, acute pancreatitis was the first manifestation of the infection with leptospira followed by septic shock. Commonly, pancreatitis does not represent the initial symptom but is rather observed as a concomitant condition, e.g. in patients with ARDS caused by leptospirosis. Leptospirosis is thought to cause systemic vasculitis in which inflammation of the vascular walls may be a consequence of the direct invasion of the infectious agent or of immune mechanisms such as immune complex deposition, auto-antibodies or cell-mediated immunity $[19,21,22]$. The mechanism of acute pancreatitis in leptospirosis still remains unclear; however, 


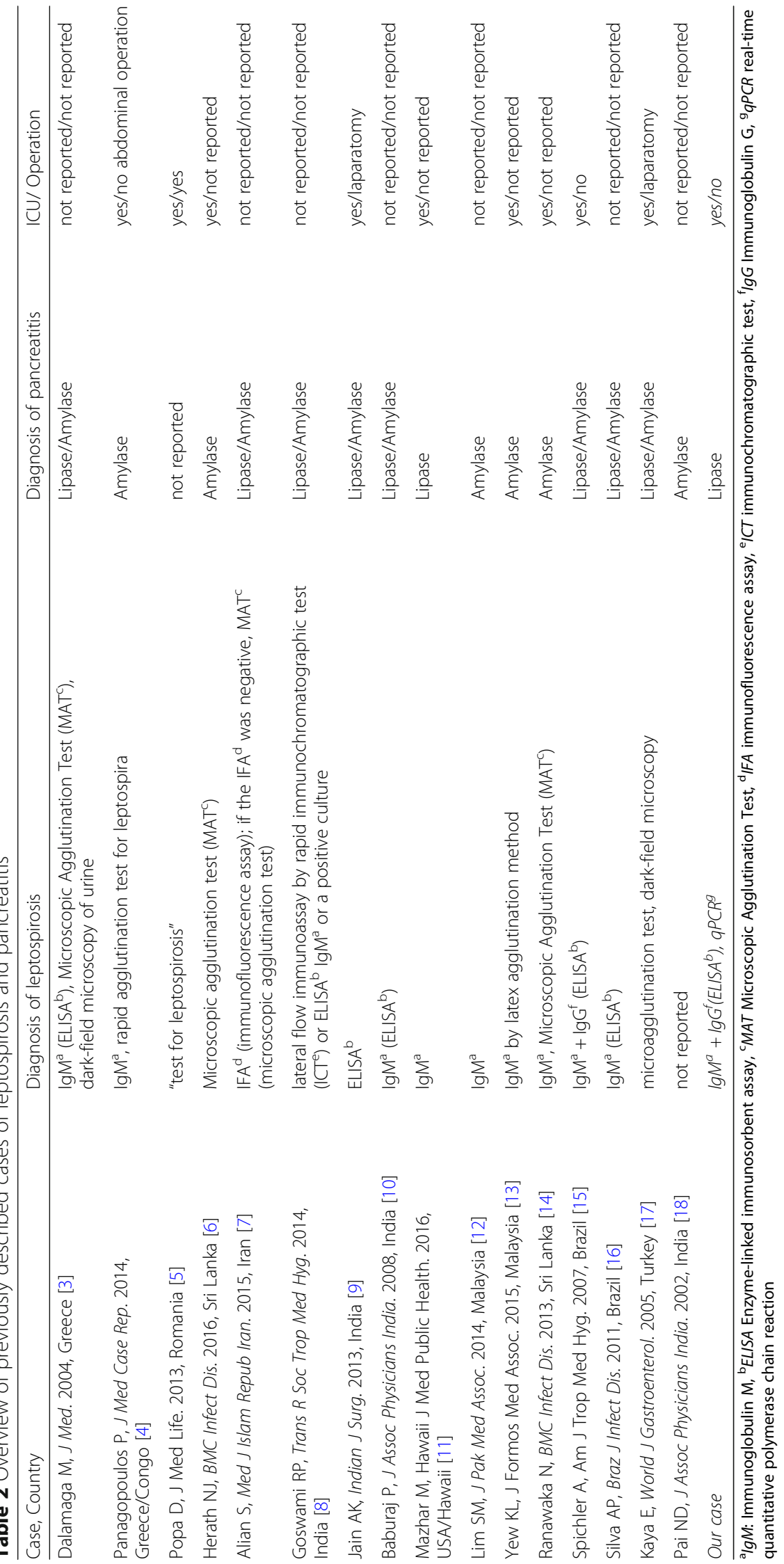


activation of proteolytic enzymes and auto-digestion causing ischemic injury and vasculitis is a probable scenario [10].

Although the reported incidence of pancreatitis in leptospirosis is infrequent, pancreatic involvement may be more common in clinical reality. Thus, due to the fulminant clinical course of our patient with multi-organ failure, we suggest that screening for leptospirosis should be included rather early in the differential diagnosis of acute pancreatitis if other typical causes have been ruled out.

An acute pancreatitis caused by an infection with Leptospira interrogans is a very rare but potentially life-threatening disease. Serologic tests and antibiotics for leptospirosis should be considered, if no other reasons for pancreatitis can be identified.

\section{Abbreviations}

ANA: Antinuclear antibody; ANCA: Anti-neutrophil cytoplasmatic antibody; ARDS: Acute respiratory distress syndrome; DNA: Deoxyribonucleic acid; ED: Emergency department; ERCP: Endoscopic retrograde cholangiopancreatography; ICU: Intensive care unit; MRSA: Methicillinresistant Staphylococcus aureus; PCR: Polymerase chain reaction

\section{Acknowledgements}

Not applicable.

\section{Funding}

The article processing charge was funded by the German Research Foundation (DFG) and the University of Freiburg in the funding programme Open Access Publishing. The funding source had no influence in the design of the study and collection, analysis, and interpretation of data and in writing the manuscript.

\section{Availability of data and materials}

Data collection and sharing is not applicable to this article as no datasets were generated or analyzed during the current study.

\section{Authors' contributions}

AM, RK, RT and TB treated the patient, made the clinical diagnosis, and wrote and revised the manuscript. All authors have read and approved the manuscript.

\section{Ethics approval and consent to participate}

Not applicable. The described patient provided written consent to publish this case report.

\section{Consent for publication}

The described patient provided written consent to publish this case report.

\section{Competing interests}

The authors declare that they have no competing interests

\section{Publisher's Note}

Springer Nature remains neutral with regard to jurisdictional claims in published maps and institutional affiliations.

\section{Author details}

'Department of Cardiology and Angiology I, Heart Center Freiburg University, Faculty of Medicine, University of Freiburg, Freiburg, Germany. ${ }^{2}$ Department of Medicine II, Medical Center, Faculty of Medicine, University of Freiburg, Hugstetter Strasse 55, 79106 Freiburg, Germany.
Received: 14 February 2019 Accepted: 26 April 2019

Published online: 08 May 2019

References

1. Robert Koch-Institut. RKI-Ratgeber für Ärzte - Leptospirose. Epidemiologisches Bulletin, Robert Koch-Institut, Epidemiologie und Gesundheitsberichterstattung. 19. Oktober 2015 / Nr. 42. DOI https://doi.org/10.17886/EpiBull-2015-012

2. Schuller S, Francey T, Hartmann K, Hugonnard M, Kohn B, Nally JE, Sykes J. European consensus statement on leptospirosis in dogs and cats. J Small Anim Pract. 2015;56(3):159-79.

3. Dalamaga M, Chavelas C, Kostoula A, Matekovits H, Karmaniolas K, Liatis S, Migdalis I. Leptospirosis presenting as acute pancreatitis and cholecystitis. J Med. 2004;35(1-6):181-5.

4. Panagopoulos P, Terzi I, Karanikas M, Galanopoulos N, Maltezos E. Myocarditis, pancreatitis, polyarthritis, mononeuritis multiplex and vasculitis with symmetrical peripheral gangrene of the lower extremities as a rare presentation of leptospirosis: a case report and review of the literature. J Med Case Rep. 2014;8:150.

5. Popa D, Vasile D, Ilco A. Severe acute pancreatitis - a serious complication of leptospirosis. J Med Life. 2013;6(3):307-9.

6. Herath NJ, Kaburapola CJ, Agampodi SB. Severe leptospirosis and pancreatitis; a case series from a leptospirosis outbreak in Anuradhapura district, Sri Lanka. BMC Infect Dis. 2016;16(1):644.

7. Alian S, Davoudi A, Najafi N, Ghasemian R, Ahangarkani F, Hamdi Z. Clinical and laboratory manifestation and outcome of icterohemorrhagic leptospirosis patients in Northern Iran. Med J Islam Repub Iran. 2015;29:308.

8. Goswami RP, Goswami RP, Basu A, Tripathi SK, Chakrabarti S, Chattopadhyay I. Predictors of mortality in leptospirosis: an observational study from two hospitals in Kolkata, eastern India. Trans R Soc Trop Med Hyg. 2014;108(12): 791-6.

9. Jain AK, Mohan LN. Acute necrotising pancreatitis associated with leptospirosis-a case report. Indian J Surg. 2013;75(3):245-6.

10. Baburaj P, Antony T, Louis F, Harikrishnan BL. Acute abdomen due to acute pancreatitis--a rare presentation of leptospirosis. J Assoc Physicians India. 2008;56:911-2.

11. Mazhar M, Kao JJ, Bolger DT Jr. A 23-year-old man with leptospirosis and acute abdominal pain. Hawaii J Med Public Health. 2016:75(10):291-4.

12. Lim SM, Hoo F, Sulaiman WA, Ramachandran V, Siew-Mooi C. Acute necrotising pancreatitis and acalculous cholecystitis: a rare presentation of leptospirosis. J Pak Med Assoc. 2014;64(8):958-9.

13. Yew KL, San Go C, Razali F. Pancreatitis and myopericarditis complication in leptospirosis infection. J Formos Med Assoc. 2015;114(8):785-6.

14. Ranawaka N, Jeevagan $V$, Karunanayake $P$, Jayasinghe $S$. Pancreatitis and myocarditis followed by pulmonary hemorrhage, a rare presentation of leptospirosis- a case report and literature survey. BMC Infect Dis. 2013;13:38.

15. Spichler A, Spichler E, Moock M, Vinetz JM, Leake JA. Acute pancreatitis in fatal anicteric leptospirosis. Am J Trop Med Hyg. 2007;76(5):886-7.

16. Silva AP, Burg LB, Locatelli JF, Manes J, Crispim M. Leptospirosis presenting as ascending progressive leg weakness and complicating with acute pancreatitis. Braz J Infect Dis. 2011;15(5):493-7.

17. Kaya E, Dervisoglu A, Eroglu C, Polat C, Sunbul M, Ozkan K. Acute pancreatitis caused by leptospirosis: report of two cases. World J Gastroenterol. 2005;11(28):4447-9.

18. Pai ND, Adhikari P. Painless pancreatitis: a rare manifestation of leptospirosis J Assoc Physicians India. 2002;50:1318-9.

19. Daher Ede F, Brunetta DM, de Silva Júnior GB, Puster RA, Patrocínio RM. Pancreatic involvement in fatal human leptospirosis: clinical and histopathological features. Rev Inst Med Trop Sao Paulo. 2003;45(6):307-13.

20. Edwards CN, Evarard CO. Hyperamylasemia and pancreatitis in leptospirosis. Am J Gastroenterol. 1991;86(11):1665-8

21. Lau C, Smythe L, Weinstein P. Leptospirosis: an emerging disease in travellers. Travel Med Infect Dis. 2010;8:33-9.

22. Plank R, Dean D. Overview of the epidemiology, microbiology, and pathogenesis of Leptospira spp. in humans. Microbes Infect. 2000;2:1265-76. 